\title{
Special issue on engineering applications of neural networks
}

\author{
Elias Pimenidis ${ }^{1} \cdot$ Chrisina Jayne $^{2}$ \\ Received: 9 May 2020 / Accepted: 9 May 2020 / Published online: 23 May 2020 \\ (C) Springer-Verlag London Ltd., part of Springer Nature 2020
}

Neural Computing has been at the forefront of the resurgence of artificial intelligence in the first two decades of the twenty-first century. Technology and industrial applications have seen considerable benefits from neural computing applications and will continue to do so in the foreseeable future. Other disciplines such as medicine are rapidly capitalising on the capabilities that $\mathrm{AI}$ has to offer. This special issue on Engineering Applications of Neural Networks offers nine papers that address a wide range of application areas that could deliver considerable benefits to industry and society.

In their work titled "Single and ensemble classifiers for defect prediction in sheet metal forming under variability “, Dib, N. J. Oliveira, Marques, M. C. Oliveira, Fernandes, Ribeiro, and Prates, explore machine learning techniques to predict the occurrence of defects in sheet metal forming processes. An empirical analysis of performance of ML techniques considering both single learning and ensemble models is performed to explore the accuracy of predictions in cases where defects are dependent on exposure to sources of scatter in the material properties and process parameters.

On a more socially oriented utilisation of AI, Gupta, McClatchey, and Caleb-Solly, investigate the utility of unsupervised machine learning and data visualisation for tracking changes in user activity over time. Analysing unlabelled data generated from passive and ambient smart home sensors, such as motion sensors, for activity recognition, can lead to practical methods that can provide meaningful information to support timely interventions

Elias Pimenidis

Elias.Pimenidis@uwe.ac.uk

Chrisina Jayne

C.Jayne@tees.ac.uk

1 University of the West of England, Frenchay Campus, Coldharbour Lane, Bristol BS16 1QY, UK

2 Teesside University, Middlesbrough, Tees Valley TS1 3BX, UK based on changing user needs. This can be particularly useful to carers that cannot be available on a twenty-four hour basis, but could be enabled to provide an enhanced service based on appropriate and timely intervention in response to predicted behaviour. Their work is titled, "Tracking changes in user activity from unlabelled smart home sensor data using unsupervised learning methods".

Microwave energy with its ability to rapidly provide large amounts of heat is widely used in many industrial applications. Tunnel microwave processing is successfully applied in heating or drying of wood, cement, ceramics, lignite, and food among others. The efficiency and productivity of the process depends on the right controller for it. In their efforts to develop a simple and accurate model which allows to design and implement a an efficient controller, Liu, Liang, Xiong, and Wang propose a novel hybrid algorithm to construct an improved recurrent neuron network (RNN) for modelling the tunnel microwave thermal process. Their work titled "Integrated CS optimisation and OLS for recurrent neural network in modelling microwave thermal process" shows how a hierarchical learning process, in which the recurrent neurons of RNN are optimised by the cuckoo search (CS) algorithm is employed in the design, while the effectiveness and efficiency of the model are guaranteed by using the orthogonal least squares (OLS) method.

In their paper titled "Robust anomaly identification algorithm for noisy signals: spacecraft solar panels model", Murtada and Omran introduce their work on developing an efficient and robust technique for fault detection and identification in noisy environments. This is of particular importance to one of the most vital subsystems of spacecraft that of electrical power subsystem (EPS). The research presents a robust, generalised and efficient FDI technique using STE-Prony scheme for extracting distinct features from noisy signals that are related to spacecraft solar panel anomalies. The proposed scheme is combined with FFNN classifier that produces a robust FDI technique for classification over other noise-sensitive techniques. The authors claim $100 \%$ accuracy for identifying all types of 
anomalies in noisy environments at noise amplitude levels up to $30 \%$ of the signal amplitude.

Polatidis, Papaleonidas, Pimenidis, and Iliadis, attempt to address the reproducibility of results as this is encountered in recommender systems' evaluation when using different offline evaluation libraries. Their work titled "An explanation-based approach for experiment reproducibility in recommender systems" proposes an approach that is based on explanations that can be used to assist researchers in reproducing the results of an experimental evaluation. The impact of such research can have a considerable bearing in different domain areas where critical systems such as medical systems and autonomous vehicles are evaluated offline.

Swarm intelligence is about developing collective behaviours to solve complex, ill-structured and large-scale problems. In "A honeybees-inspired heuristic algorithm for numerical optimisation" Dugenci and Aydin, present a hybrid bee algorithm which harmonises bee operators of two mainstream well-known swarm intelligence algorithms inspired of natural honeybee colonies. The efficiency of the hybrid algorithm is demonstrated in comparison with the parent algorithms in solving two types of numerical optimisation problems; functional optimisation benchmarks, and ANN training through optimising the weights of connection links of feed-forward NN models.

Video tampering detection remains an open problem in the field of digital media forensics. This is the focus of the research presented by Johnston, Elyan, and Jayne in "Video tampering localisation using features learned from authentic content". Deep learning methods have already shown great promise in discovering effective features from data, particularly in the image domain, but labelled datasets of varied, state-of-the-art, tampered video which are large enough to facilitate machine learning do not exist. The authors used convolutional neural networks to estimate quantisation parameter, deblock setting and intra/inter mode of pixel patches from an H.264/AVC sequence, to address the problem.

Vandersmissen, Knudde, Jalalvand, Couckuyt, Dhaene, and De Neve, propose a novel approach towards automatic indoor human activity recognition, using deep neural networks that take as input data originating from radar and video camera sensors. In "Indoor human activity recognition using high-dimensional sensors and deep neural networks" they present and utilise two data sets that they have constructed consisting of samples distributed over six different types of gestures and events. They claim to have successfully built a solution to automatically recognise gestures and events in a realistic scenario, taking advantage of both a frequency-modulated continuous-wave (FMCW) radar and a video camera sensor.

"A novel recommendation method based on general matrix factorisation and artificial neural networks", written by Kapetanakis, Polatidis, Alshammari, and Petridis, presents an approach to address the limitations in terms of accuracy recommendations stemming from high-frequency low-relevance user-item bias, data specificities and individual user patterns and needs that may be hidden in data. The authors propose a novel recommendation approach that improves accuracy and requires significantly less maintenance compared to traditional collaborative filtering.

The guest editors would like to thank all authors for their efforts and significant contributions that we expect will provide useful insights to the scientific community.

Finally, we express our gratitude to the Editor in Chief, Professor John Macintyre, for the opportunity and trust in us editing this special issue on Engineering Applications of Neural Networks.

The Guest Editors

Dr Elias Pimenidis and Professor Chrisina Jayne

Publisher's Note Springer Nature remains neutral with regard to jurisdictional claims in published maps and institutional affiliations. 